\title{
Ligne d'équilibre des glaciers: le stade de référence de 1850 dans les Alpes calcaires occidentales
}

\section{Introduction}

\subsection{La ligne d'équilibre de 1850 - référence pour le Tardiglaciaire}

La méthode classique d'étude des stades tardiglaciaires implique en général la reconstitution géométrique des divers stades glaciaires et le calcul pour chacun d'eux de l'abaissement de la ligne d'équilibre («Schneegrenzdepression»). Cet abaissement est calculé par rapport au dernier maximum historique, vers 1850 , qui est considéré comme le dernier stade d'équilibre à peu près synchrone pour tous les glaciers alpins. Le stade de 1850 constitue ainsi le stade de référence pour toutes les reconstitutions paléoclimatiques (GROSS et al. 1977).

Cette méthode a permis l'établissement d'un modèle de déglaciation et de nombreuses corrélations surtout dans les Alpes orientales et centrales (MAISCH 1981, 1982, 1987). Dans les Alpes occidentales par contre, les études sont encore peu nombreuses (AESCHLIMANN 1983, BLESS 1984, DORTHE-MONACHON 1986, WETTER 1987), et les corrélations avec le modèle des Alpes orientales se révèlent problématiques. L'une des principales difficultés auxquelles se heurte l'utilisation de la méthode classique est l'absence de glaciers actuels dans de nombreuses vallées des Alpes calcaires, ainsi que dans toutes les Préalpes. L'altitude de référence de 1850 doit alors être recherchée dans une vallée voisine. $\mathrm{Vu}$ que le calcul doit tenir compte de l'orientation de la vallée, le site de référence doit parfois être cherché assez loin, et le choix d'un glacier plutôt qu'un autre devient alors difficile et souvent arbitraire.

\subsection{Une référence par rapport au réchauffement récent}

Le stade de 1850 marque également la fin du «Petit âge glaciaire», dont il représente le dernier maximum. Il est donc également le stade de référence pour mesurer le retrait des glaciers consécutif à la tendance au réchauffement enregistrée depuis bientôt 150 ans. Ce signal de l'évolution climatique récente a été étudié en détail aux Grisons par MAISCH $(1987 \mathrm{a}+\mathrm{b}, 1988,1992)$ et fera l'objet, dans les années à venir, d'un projet de recherche à l'échelle de la Suisse entière. Nous ne développerons donc pas cet aspect dans le présent article.

\subsection{Le problème des variations géographiques de la ligne d'équilibre}

La question des variations d'altitude régionales de la ligne d'équilibre des glaciers et de l'existence de tendances ouest-est et nord-sud (externe-interne) dans les Alpes suisses a été abordée par JEGERLEHNER (1902) déjà, plus récemment par MÜLLER et al. (1976). La tendance à l'élévation de la ligne d'équilibre du front occidental vers les massifs internes de l'arc alpin est un fait maintenant bien connu, et qui s'explique par l'effet de barrage du relief sur les précipitations et la diminution de l'humidité du climat vers l'intérieur de la chaîne.

Une tendance moins nette, et moins régulière, à l'élévation de la ligne d'équilibre semble se dessiner de l'ouest vers l'est. Elle pourrait résulter de la continentalité croissante du climat vers l'est, générale pour tout le continent européen. Dans les Alpes romandes, la carte de MÚLLER et al. (1976) montre un gradient très fort du SW au NE, qui semble cependant difficilement explicable par une tendance climatique générale.

\subsection{Vers une référence régionale}

Ces raisons nous ont amenés à bâtir une référence régionale valable pour tout le front occidental des Alpes suisses. La région considérée comprend les massifs calcaires des nappes de Morcles-Doldenhorn et du Wildhorn (sens large, selon ESCHER et al. 1988 et STECK et al. 1989), du Balmhorn au Mont Ruan (fig. 1). Nous avons pris en compte tous les appareils figurant à l'inventaire suisse des glaciers (MÜLLER et al.), ainsi que quelques glaciers aujourd'hui disparus, mais dont l'existence en 1850 a pu être attestée.

\section{Méthode}

Pour reconstituer l'extension des glaciers vers 1850, nous avons utilisé en particulier les levés originaux de la carte Dufour. Dans la région considérée, ces levés topographiques ont été réalisés entre 1839 et 1841 (soit une dizaine d'années avant l'extension maximale des glaciers), sauf

Claire Dorthe-Monachon, Philippe Schoeneich, Institut de Géographie, Université de Lausanne, BFSH 2, 1015 Lausanne 


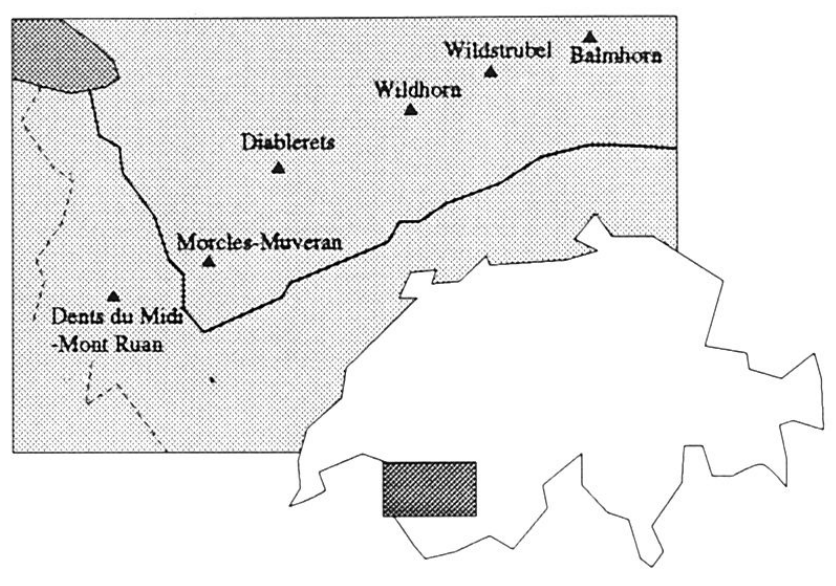

Fig.1 Carte de situation des massifs étudiés. pour la région Dents du Midi-Mont Ruan, levée en 1857 seulement. Les minutes de terrain sont dessinées en courbe de niveau, à l'échelle 1: 50 000. Si l'identification des limites des glaciers semble correcte dans la plupart des cas, le dessin du relief présente de très fortes distorsions dans les zones de montagne par rapport à la carte actuelle. Par ailleurs l'équidistance inhabituelle de $30 \mathrm{~m}$, l'absence de courbes de niveau maîtresses et les fréquentes inadéquations entre points cotés et courbes de niveau rendent difficile un repérage précis des altitudes. A cela s'ajoute que sur les glaciers les courbes de niveau ne respectent pas l'équidistance (la topographie des zones de rocher et de glace étant très certainement dessinée à distance).

Pour toutes ces raisons, les calculs de surface n'ont pas été faits d'après les levés originaux, mais les stades ont été reportés sur la carte au 1:25000 actuelle (fig. 2). L'exten-

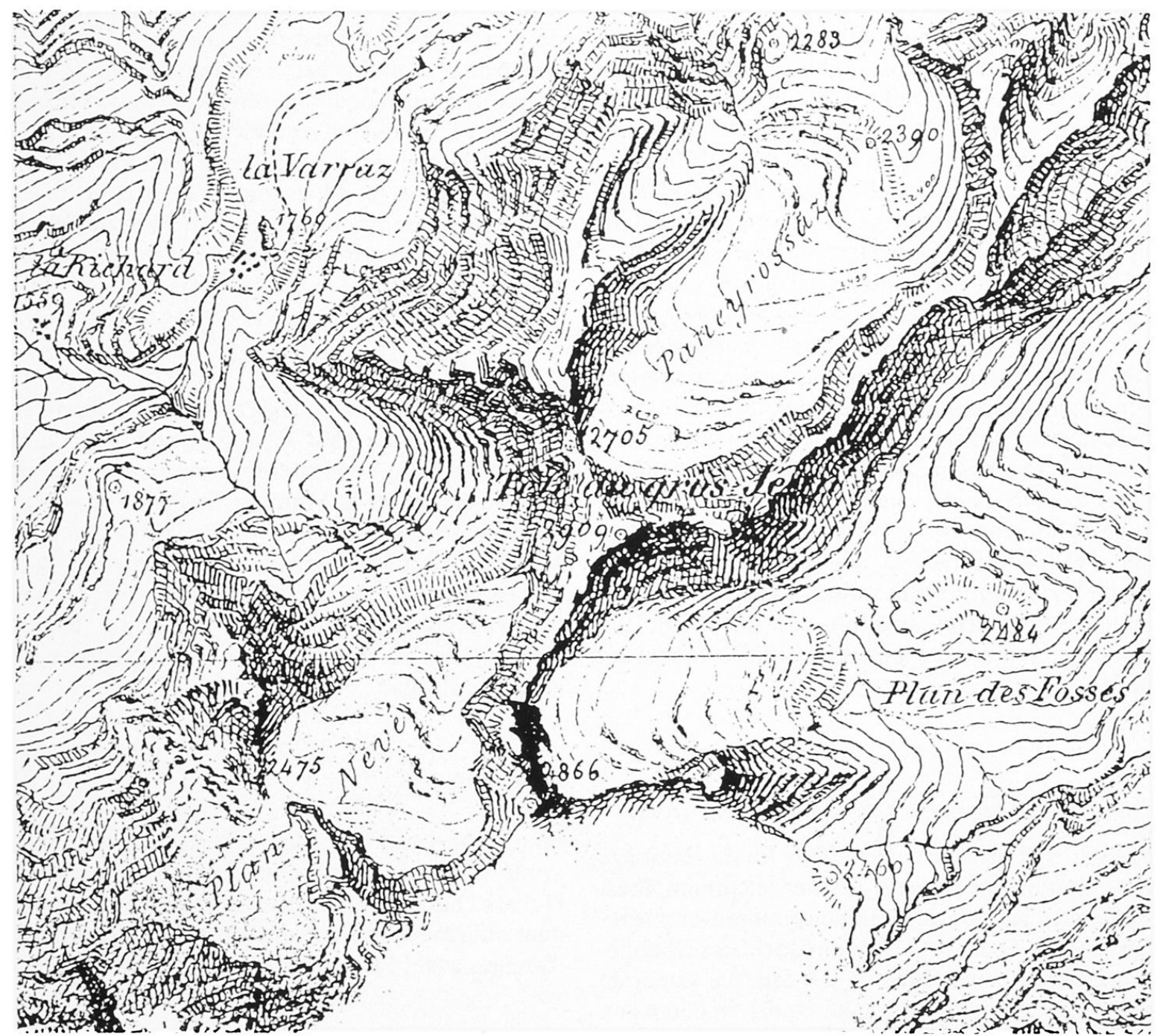

Fig. 2 Le passage du levé original à la carte nationale (reproduit avec l'autorisation de l'Office fédéral de topographie du 29 janvier 1993):

a) Extrait du levé original no 391a, levé en 1939 par Wolfsberger, échelle originale 1:50 000, équidistance $30 \mathrm{~m}$. 
sion des glaciers a été confrontée à toutes les données disponibles: carte Siegfried (dont la première édition donne une bonne image du stade de 1890), photos aériennes, cartes géologiques, études géomorphologiques et pour certains cas des levés de terrain personnels. Nous disposions de levés géomorphologiques récents de SCHOEN. EICH (en préparation) et de REYNARD (1992) pour le massif des Diablerets, de MONACHON (1978) et REYNARD (1992) pour le massif du Wildhorn, de DORTHE-MONA. CHON (1993) et STUBER (1989) pour les massifs du Wildstrubel et du Balmhorn, et de PHILLIPS (1993) pour les Dents de Morcles. Pour les Dents du Midi et le Mont Ruan la reconstitution s'appuie sur les seuls documents cartographiques.

Dans la plupart des cas ce mode de faire a permis de localiser avec certitude les moraines de 1850 et de reconstituer avec une bonne fiabilité les limites des glaciers de l'époque.
La topographie de surface des glaciers a ensuite été redessinée en s'inspirant des courbes de niveau des levés originaux de la carte Dufour, puis les surfaces ont été digitalisées. La ligne d'équilibre a été calculée selon la méthode classique de l'AAR, avec un rapport standard de 0,67 (rapport 2/1, «Flächenteilungsmethode», GROSS et al. 1977).

La validité du résultat a été vérifiée par rapport à l'altitude de départ des moraines latérales («Moränenansatzmethode»): du point de vue théorique, des moraines latérales ne peuvent se former que dans la zone d'ablation, c'est-à-dire à partir du moment où les lignes de flux de la glace se dirigent vers la surface du glacier et amènent le matériel vers sa bordure (LICHTENECKER 1936). Là où elles existent, les moraines latérales donnent donc une altitude minimum pour la ligne d'équilibre, et permettent de tester la cohérence du résultat calculé.

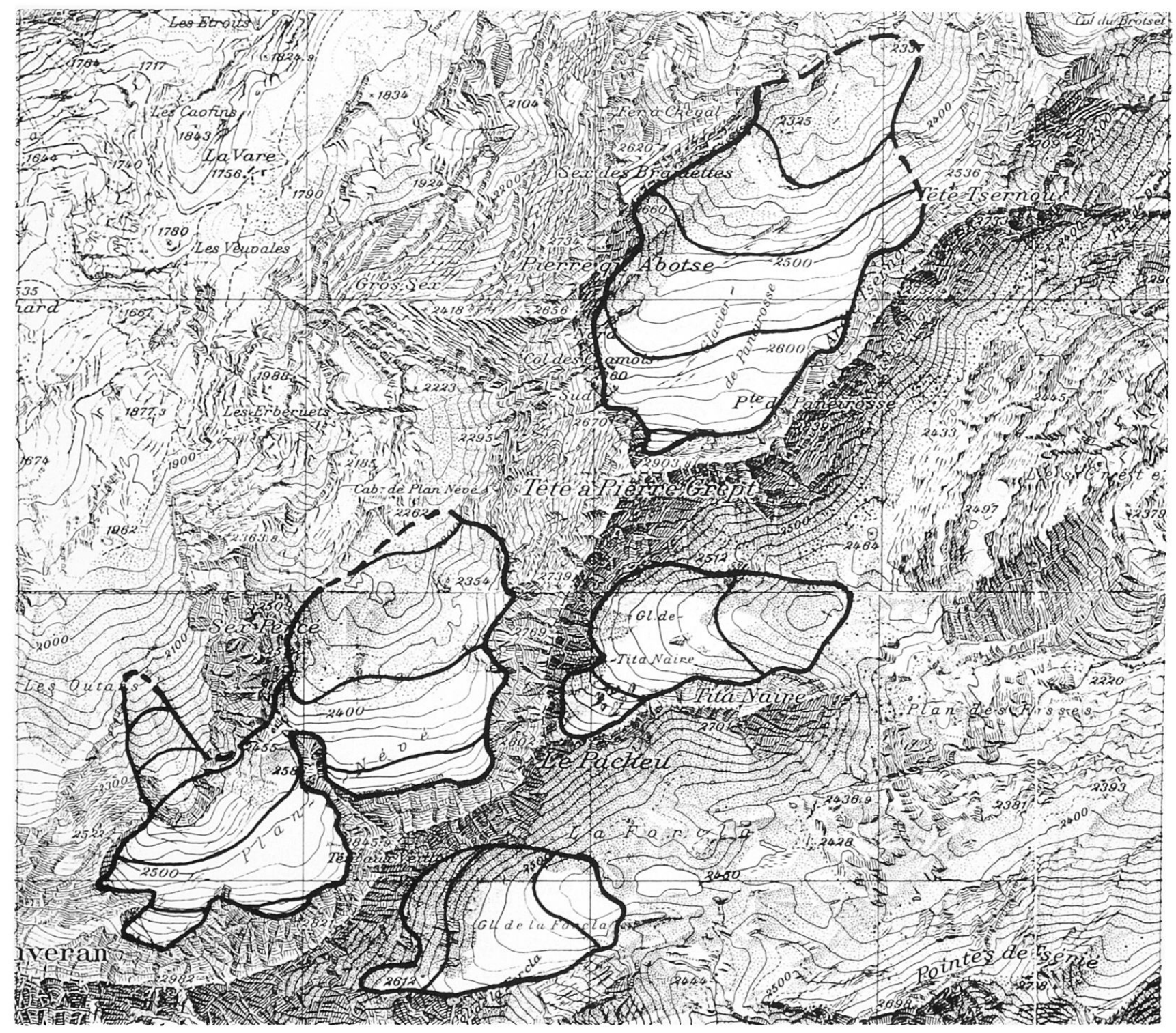

Fig. 2 b) Report sur la carte nationale des limites des glaciers et reconstitution des courbes de niveau directrices. 
Les résultats ont été interprétés en tenant compte de l'orientation des glaciers, selon 8 secteurs. Ils ont été représentés d'abord massif par massif, et comparés en vue de mettre en évidence une éventuelle tendance spatiale, puis regroupés pour la construction du modèle régional.

\section{Résultats}

\subsection{Massifs des Diablerets, du Wildhorn et du Wildstrubel}

Ces trois massifs, formés tous trois par la nappe du Wildhorn, ont une morphologie et par conséquent un style d'englacement semblables: grands glaciers relativement plats reposant sur des dalles calcaires en dip-slope à faible pendage, cirques peu encaissés souvent englacés jusqu'aux arêtes, sommets couverts de calottes, langues glaciaires larges et courtes.

Les résultats sont représentés dans la fig. 3, et donnent dans l'ensemble un motif de répartition très cohérent. Seuls quelques appareils s'en écartent.

Dans le massif des Diablerets, le glacier des Diablerets et le glacier de la Tchiffa, qui sont des appareils respectivement suspendu et régénéré, donnent des valeurs climatiquement non significatives et ont par conséquent été écartés du modèle régional. Un petit appareil accroché aux flancs de l'Oldenhorn présente une valeur nettement «trop» élevée et était probablement au moins partiellement suspendu.

Dans le massif du Wildhorn, le glacier des Eaux Froides, suspendu, a également été écarté du modèle régional. Le glacier des Audannes-Ténéhet, considéré comme un seul appareil dans l'inventaire, a été séparé, ce qui est plus conforme à la dynamique d'écoulement. Le glacier de Ténéhet était probablement aussi partiellement suspendu, et présente de ce fait une altitude de la ligne d'équilibre plus élevée que le glacier de Zanfleuron, de morphologie et d'orientation semblables. Le Tungelgletscher a été également séparé en deux parties, le Tungelgletscher proprement dit et le Chilchligletscher, quoique la séparation des zones d'alimentation soit ici très difficile.

Les glaciers du massif du Wildstrubel, malgré leur extrême diversité de taille, de forme et d'exposition, montrent des résultats peu étalés, compris entre 2585 et 2820 m (fig. 4). Le glacier de Plaine Morte, dont la surface équivaut à près de la moitié de la surface englacée du massif, a une ligne d'équilibre à peine plus élevée que la moyenne. Cela pourrait provenir de la morphologie très plate de l'ensemble, qui tend à minimiser l'influence de l'exposition.

\subsection{Massif du Muveran, des Dents de Morcles, des Dents du Midi et du Mont Ruan}

Ces quatre massifs appartiennent à la nappe de Morcles et présentent entre eux une morphologie semblable, mais très différente de celle des trois massifs précités: ils sont beaucoup plus aérés et découpés en une série de dents plus ou moins isolées. De ce fait, les glaciers atteignent rarement les arêtes et les sommets et ils s'étendent au pied de hautes parois rocheuses.

Cette différence de style d'englacement se traduit par une différence très nette de l'altitude de la ligne d'équilibre des glaciers, qui se trouve en moyenne 200 à 300 m plus bas que dans la nappe du Wildhorn (fig. 5).

Dans les Dents du Midi, le grand glacier de Plan Névé, très peu encaissé, donne une valeur nettement plus haute, et proche de celle du glacier des Audannes, de morphologie et d'orientation semblables. Comme ce dernier, une partie de son front était probablement suspendue.

Dans le massif du Mont Ruan-Tour Sallière, le glacier Noir, formé d'une partie suspendue et d'un cône régénéré, donne une valeur climatiquement non significative et doit être écarté du modèle régional. Le glacier des Rosses, au front suspendu, donne une valeur surestimée. L'absence de documents cartographiques anciens sur la partie française n'a malheureusement pas permis de calculer les lignes de névés des glaciers du Ruan et de Prazon. L'absence quasi totale de moraines ne permet pas leur reconstitution à postériori (NOVERRAZ 1970).

\subsection{Massif du Balmhorn}

Si, géologiquement, les environs du Balmhorn se rattachent aux massifs de Morcles et des Dents du Midi, l'englacement y prend pourtant des formes très différentes. Le sommet du Balmhorn, englacé sur toutes ses faces, est le point culminant de toute la région considérée dans cette étude. L'ensemble du massif est profondément découpé et des glaciers de vallée (Schwarzgletscher, Balmhorngletscher) complexes et à forte dénivellation en occupent les pentes. La forme de ces appareils rappelle, toutes proportions gardées, celle de certains grands glaciers du sud du Rhône.

Les altitudes obtenues sont très proches de celles du Wildstrubel (fig. 4). Le glacier de l'Altels et le glacier ouest du Rinderhorn sont des appareils de versant plaqués sur un dip-slope à pente forte et constante. L'éboulement régulier du front est probablement responsable de l'altitude particulièrement élevée de leurs lignes d'équilibre, proches de $3000 \mathrm{~m}$, qui ont été écartées du modèle régional.

\section{Le modèle régional}

Les résultats montrent que les massifs étudiés peuvent être séparés en deux groupes, liés respectivement à la nappe du Wildhorn et à celle de Morcles: dans ces derniers, l'altitude de la ligne de névé de 1850 est en moyenne 200 à $300 \mathrm{~m}$ plus bas que dans les premiers. Nous pensons que cette différence ne doit pas être interprétée comme une tendance climatique régionale est-ouest, mais qu'elle résulte d'une différence dans le 


\section{Massif des Diablerets}

$\mathrm{N}$

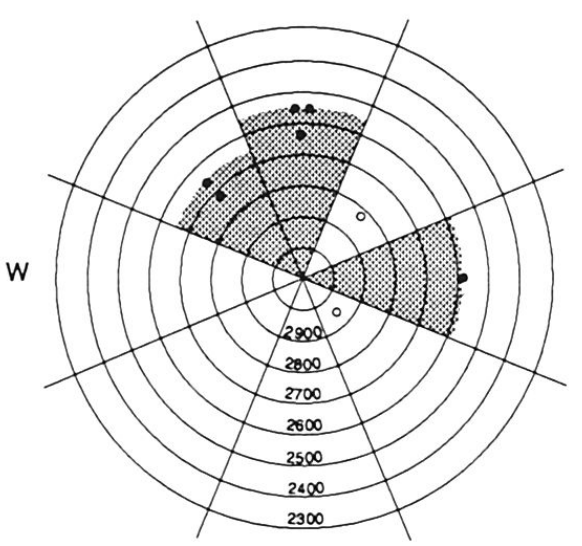

$\mathrm{S}$
N

E W

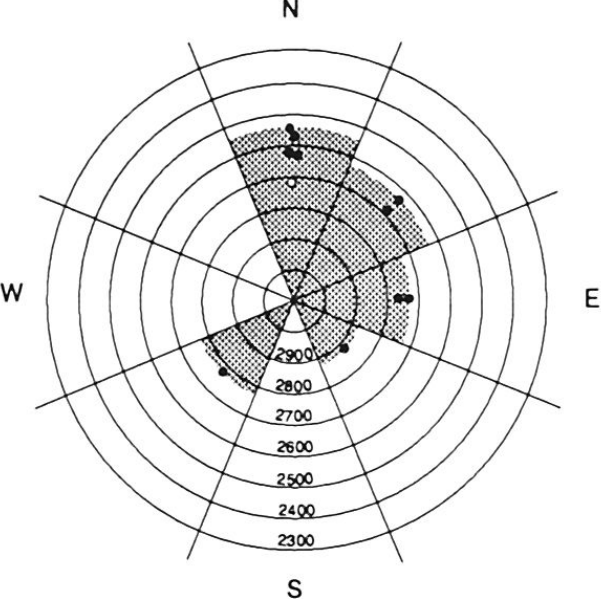

$\mathrm{S}$

- glaciers suspendus

- tous autres glaciers

\begin{tabular}{|c|l|c|c|l|}
\hline \multicolumn{6}{|l|}{ Glaciers du massif des Diablerets } \\
\hline Code inv. & Nom & Orientation & LE 1850 & Remarques \\
\hline B $16 / 1+2$ & Glaciers du Dar et Gl. du Sex Rouge & N & 2570 & formaient un seul appareil \\
B $16 / 3$ & Glacier de Prapio & NW & 2715 & \\
B $16 / 4$ & Mauvais Glacier & NW & 2670 & \\
B $16 / 5$ & Glacier de Pierredar & N & 2570 & \\
B $16 / 6+7$ & Glacier de Culan & N & 2635 & \\
B 21/5+7 & Glacier des Diablerets + Tchiffa & SE & 2940 & glaciers suspendu + régénéré \\
B 22/1 & Glacier de Zanfleuron & E & 2590 & \\
& flanc du Mont Brun & NE & 2820 & glacier suspendu \\
\hline
\end{tabular}

\begin{tabular}{|c|l|c|c|l|}
\hline \multicolumn{4}{|l|}{ Glaciers } & du massif du Wildhorn \\
\hline Code inv. & Nom & Orientation & LE 1850 & Remarques \\
\hline A 55 / / 6 & Totetäli & NE & 2635 & \\
A 56d / 1-3 & Tungelgletscher & N & 2580 & \\
" & Chilchligletscher & NE & 2695 & \\
A 56d / 4 & Geltengletscher E & N & 2620 & \\
A 56d / 5 & Geltengletscher W & N & 2555 & \\
A 56d / 6 & Furggetäli & N & 2625 & \\
B 22 / 4 & Glacier du Brochet & SW & 2780 & \\
B 23 / 2 & Glacier des Eaux Froides & N & 2710 & suspendu \\
B 23 / 3 & Glacier de Ténéhet & E & 2730 & partiellement suspendu \\
& Gl.des Audannes & SE & 2880 & \\
B 23 / 4 & versant est du Schnidehorn & E & 2770 & \\
\hline
\end{tabular}

Fig. 3 Répartition des altitudes de ligne de névé par secteur dans les massifs des Diablerets et du Wildhorn. 


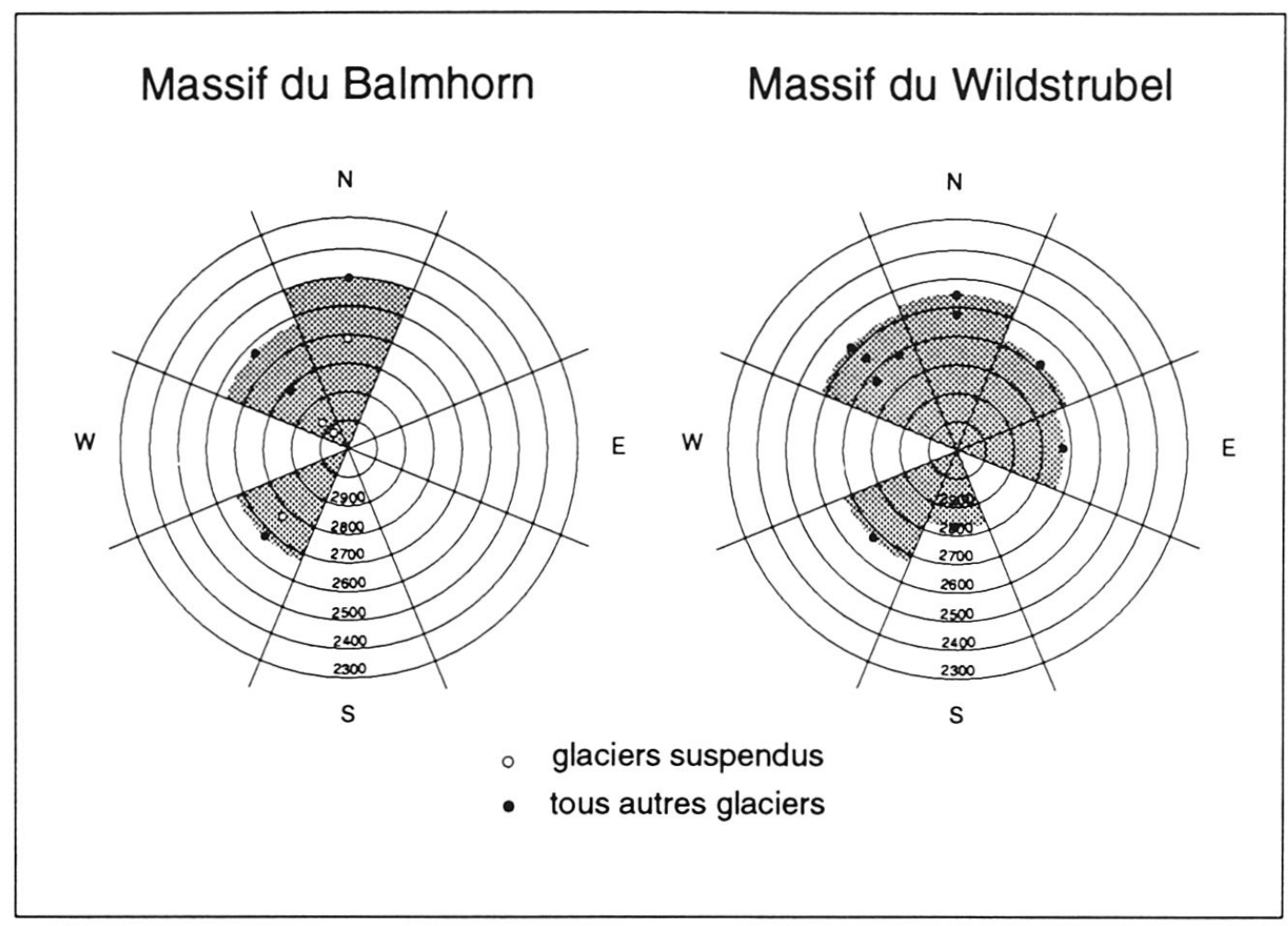

\begin{tabular}{|c|c|c|c|c|}
\hline \multicolumn{5}{|c|}{ Glaciers du massif du Wildstrubel } \\
\hline Code inv. & Nom & Orientation & LE 1850 & Remarques \\
\hline A $55 c / 9$ & Daubengletscher & $\mathrm{NW}$ & 2720 & \\
\hline A $55 c / 10-14$ & Wildstrubelgletscher & E & 2730 & \\
\hline A $55 c / 16$ & Tälligletscher & $\mathrm{N}$ & 2630 & \\
\hline A $55 f / 1$ & Ammertengletscher & $\mathrm{NW}$ & $2585^{\prime}$ & \\
\hline A $55 f / 3$ & Glacier de la Plaine Morte & NW & 2735 & \\
\hline A $55 f / 4$ & Tierberggletscher & $\mathrm{N}$ & 2560 & \\
\hline A $55 f / 5$ & Wysshorngletscher & $\mathrm{NW}$ & 2660 & \\
\hline$B 24 / 1$ & Mont Bonvin & NE & 2685 & \\
\hline B $24 / 2$ & & $\mathrm{~S}$ & 2820 & \\
\hline B $25 / 1$ & & SW & 2675 & \\
\hline
\end{tabular}

\begin{tabular}{|c|l|c|c|l|}
\hline \multicolumn{5}{|l|}{ Glaciers du massif du Balmhorn } \\
\hline Code inv. & Nom & Orientation & LE 1850 & Remarques \\
\hline A 55 b / 18 & Balmhorngletscher & N & 2505 & \\
A 55 c / 1 & flanc nord de l'Altels & N & 2705 & \\
A 55 c / 3 & flanc nord-ouest de l'Altels & NW & 2970 & suspendu \\
A 55 c / 5 & Schwarzgletscher & NW & 2640 & \\
A 55 c / 7 & flanc nord-ouest du Rinderhorn & NW & 3045 & suspendu \\
B $25 / 3$ & Fluegletscher & S & 2760 & partiellement suspendu \\
B $25 / 7$ & Majingletscher & NW & 2805 & \\
B $35 / 4$ & Dalagletscher & SW & 2680 & \\
\hline
\end{tabular}

Fig. 4 Répartition des altitudes de ligne de névé par secteur dans les massifs du Balmhorn et du Wildstrubel. 


\section{Massif des Dents du Midi Massif de Morcles}

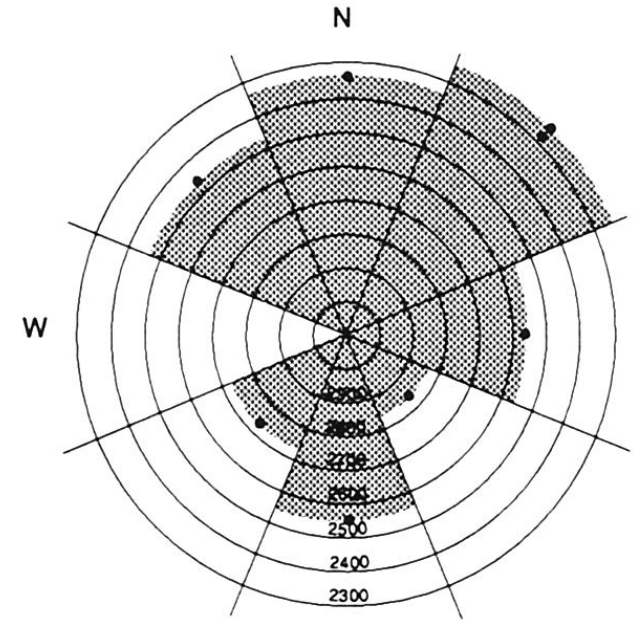

$\mathrm{S}$

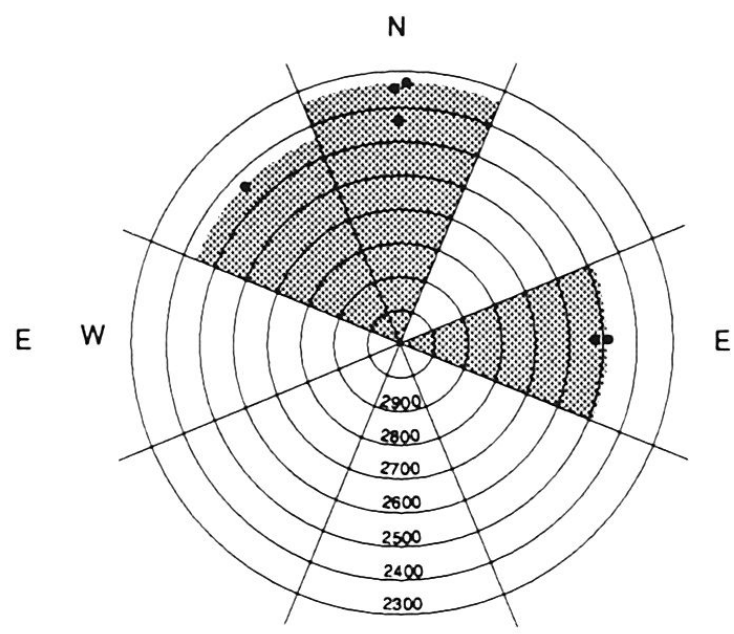

S

- glaciers suspendus

- tous autres glaciers

\section{Glaciers des massifs des Dents de Morcles et du Grand Muveran}

\begin{tabular}{|c|l|c|c|l|}
\hline Code inv. & Nom & Orientation & LE 1850 & Remarques \\
\hline B $17 / 2$ & Glacier de Paneirosse & N & 2455 & \\
B $17 / 3$ & Glacier de Plan Névé E & N & 2355 & \\
B 17 / & Glacier de Plan Névé W & NW & 2445 & \\
B 17 / & Glacier des Martinets & N & 2330 & \\
B 21/3 & Glacier de la Forcla & E & 2515 & \\
B 21/4 & Glacier de Tita Naire & E & 2495 & \\
\hline
\end{tabular}

\section{Glaciers des massifs des Dents du Midi et du Mont Ruan}

\begin{tabular}{|c|l|c|c|l|}
\hline Code inv. & Nom & Orientation & LE 1850 & Remarques \\
\hline B 92 / 3 & Glacier de la Finive & NE & 2295 & \\
B 92 / 5 & Glacier des Rosses & E & 2565 & \\
B 92 / 7 & Glacier des Fonds & S & 2540 & \\
B 92 / 9 & Glacier de la Tour Sallière & SW & 2715 & \\
B 93 / 2+3 & Glacier Noir & E & 2055 & glaciers suspendu + régénéré \\
B 93 / 5+6 & Glacier de Plan Névé & SE & 2830 & \\
B 94 / 1 & Glacier de Plan Névé & NE & 2260 & \\
B 95 / 1+2 & Glacier de Chalin & N & 2340 & \\
B 95 / 8+9 & Glacier du Mont Ruan & NW & 2475 & \\
\hline
\end{tabular}

Fig. 5 Répartition des altitudes de ligne de névé par secteur dans les massifs des Dents du Midi-Mont Ruan et de Morcles-Muveran. 

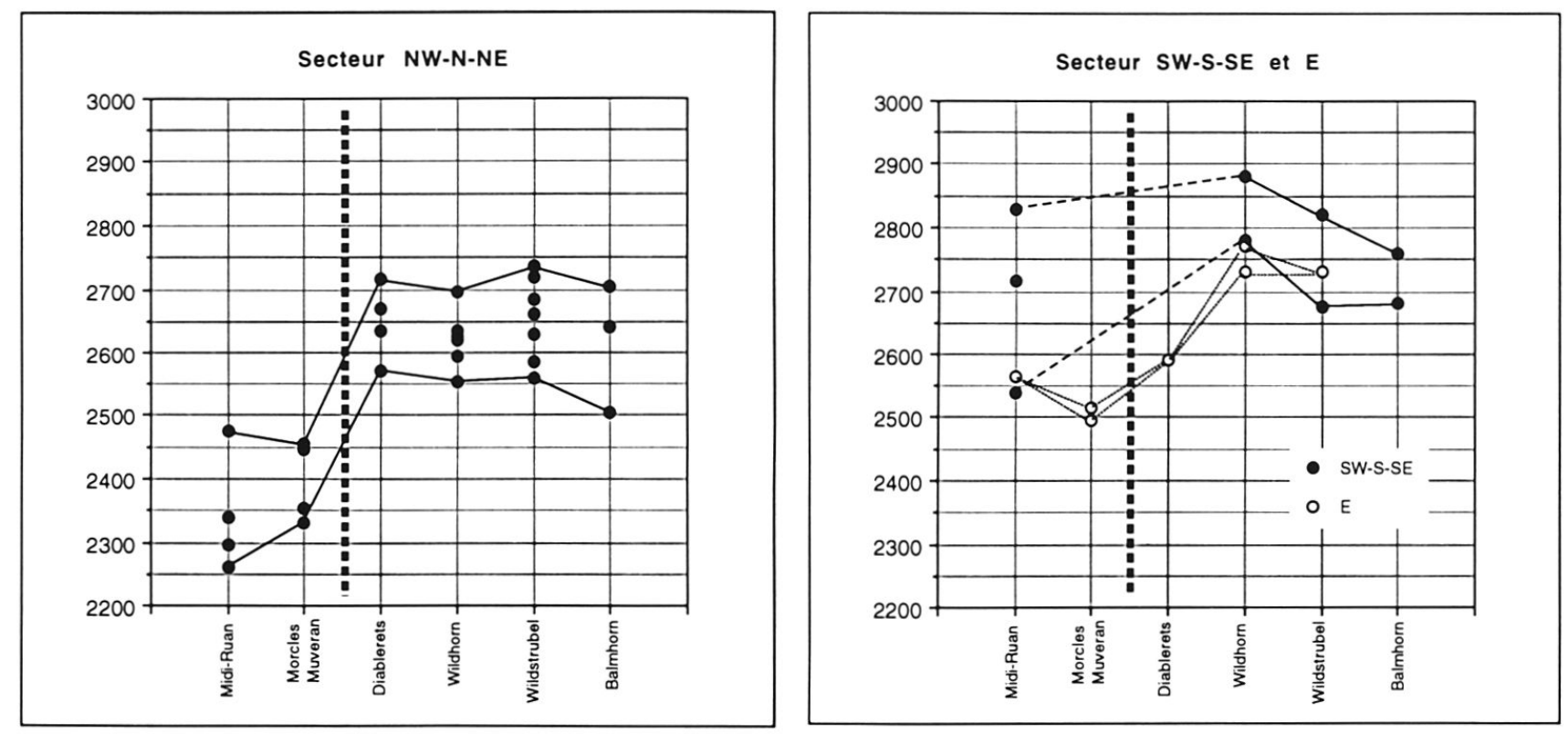

Fig. 6 Evolution de la ligne de névé d'ouest en est, pour les secteurs NW-N-NE, E et SW-S-SE.

style d'englacement des massifs, lui-même déterminé par le style tectonique différent des nappes qui les composent.

C'est ainsi que dans les massifs des Diablerets, du Wildhorn et du Windstrubel, formés par la nappe du Wildhorn, les glaciers sont en général plats et peu encaissés, et sont par conséquent alimentés essentiellement par les précipitations tombant directement sur la surface du glacier.

Dans les massifs du Muveran-Dent de Morcles, des Dents du Midi et du Mont Ruan, formés par la nappe de Morcles, les glaciers sont la plupart encaissés et dominés par de hautes parois rocheuses. Cette morphologie a une influence sur l'alimentation neigeuse des glaciers, de par l'effet sur les précipitations, et sur le transport éolien de la neige. Ces appareils sont par ailleurs alimentés pour une bonne part par des avalanches. L'effet le plus important de l'encaissement agit cependant sur l'ablation par l'effet des ombres portées. On peut noter ici que la différence d'altitude entre les deux groupes de massifs est sensible surtout dans les secteurs nord et est, alors qu'en secteur sud elle est nulle ou faible (fig. 6). Cela s'explique par le fait qu'en orientation sud l'effet d'ombre portée du relief n'existe pas. Les glaciers encaissés orientés au nord ont donc une ligne de névé comparativement plus basse que la «normale». Ils présentent souvent des assymétries liées à l'assymétrie des zones d'alimentation, et l'altitude de départ des moraines latérales ne correspond souvent pas à la ligne d'équilibre calculée. L'exemple le plus démonstratif est donné par le glacier des Martinets, au pied $\mathrm{N}$ des Dents de Morcles. Ce glacier est dominé à son sommet et sur toute la longueur de son flanc droit par une haute paroi rocheuse. La photo aérienne montre que sa ligne de névé est oblique par rapport aux courbes de niveau et qu'elle suit exactement la trace de l'ombre portée. Sa moraine latérale gauche débute très haut, presqu'au sommet du glacier, bien au-dessus de la ligne d'équilibre moyenne calculée, alors que la moraine latérale droite est presque inexistante, la zone d'alimentation se prolongeant en rive droite presque jusqu'au front (PHILLIPS 1993). Sur un tel glacier, le calcul d'une ligne d'équilibre moyenne n'a guère de sens.

Deux glaciers s'écartent de la tendance générale: le glacier des Fonds, sur le flanc sud du Mont Ruan qui, malgré son orientation, a une ligne de névé calculée très basse, et le glacier de Zanfleuron dans le massif des Diablerets. Le premier pourrait s'expliquer par l'effet d'abri du Mont Ruan sur les précipitations et par la présence d'une haute paroi sur son flanc ouest, le protégeant du soleil de l'après-midi. Le second a une ligne de névé calculée nettement plus basse que les glaciers de même orientation est et de même morphologie. Ce fait ne peut s'expliquer ni par l'encaissement ni par l'effet des précipitations ou d'un transport éolien. Peut-être faut-il en chercher la cause dans la dynamique de ce glacier très plat qui peut être assimilé, par certains aspects, à une calotte.

La comparaison avec les résultats de JEGERLEHNER (1902) est instructive. Cet auteur a utilisé essentiellement une autre méthode, selon laquelle la ligne de névé "climatique" se situerait dans une fourchette comprise entre l'altitude minimale des sommets englacés et l'altitude maximale des surfaces favorables non englacées ("Gipfelmethode»). Il obtient pour tous les massifs considérés ici des altitudes sensiblement égales, avec même une élévation aux Dents du Midi! Pour les massifs des Diablerets, du Wildhorn et du Wildstrubel, ses résultats 
concordent assez bien avec les nôtres, alors que pour toute la zone Muveran-Mont Ruan, ils sont systématiquement plus hauts. Ceci confirme encore que l'abaissement observé de l'altitude des glaciers est dû ici aux caractéristiques topographiques locales et non à une tendance climatique régionale.

Les glaciers qui sont groupés autour du sommet du Balmhorn, y compris les glaciers de Flue et de la Dala, auxquels nous avons ajouté le Majinggletscher, montrent des altitudes de la ligne d'équilibre très proches de celles des glaciers du Wildstrubel voisin malgré des caractéristiques morphologiques très particulières. Ceci peut s'expliquer par le fait que les surfaces d'alimentation de ces appareils, bien que très pentues, montent jusqu'aux arêtes, et sont donc prises en compte en totalité dans le calcul de la ligne d'équilibre.

Compte tenu de ces différences morphologiques, les résultats présentent une grande cohérence, et aucune tendance régionale ne peut être mise en évidence. Nous proposons donc un modèle de référence régional comportant: une valeur supérieure, pour les glaciers "plats» et une valeur inférieure pour les glaciers encaissés (fig. 7). Pour la première valeur, nous avons négligé les glaciers trop petits et ceux qui présentent une morphologie particulière.

Pour les calculs d'abaissement de la ligne de névé des stades tardiglaciaires dans des vallées ne comportant plus de glacier, le modèle donne ainsi des fourchettes de valeurs utilisables selon l'orientation et la morphologie

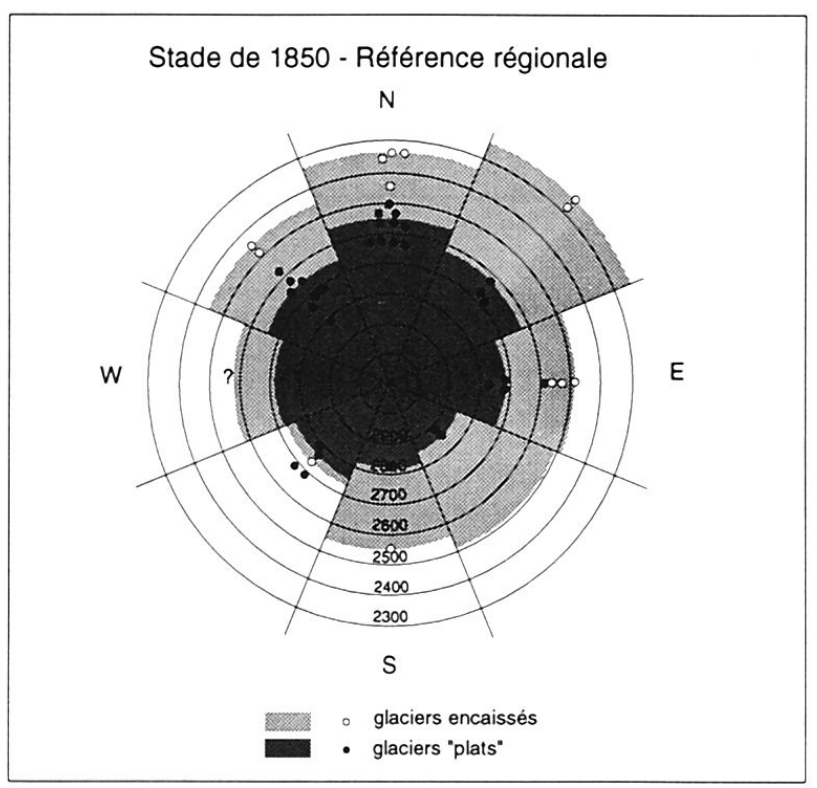

Fig. 7 Modèle de référence régional pour la ligne de névé des glaciers au stade de 1850 dans les Alpes calcaires occidentales. des cirques.

Pour les reconstitutions paléoclimatiques faisant intervenir des calculs d'isothermes et de précipitations, seule la valeur supérieure relative aux glaciers "plats» a une réelle signification climatique.

\section{Conclusions}

De nombreux auteurs ont mis en évidence des tendances régionales dans la répartition de l'altitude de la ligne de névé des glaciers dans les Alpes suisses. L'élévation de la ligne de névé du front occidental vers les vallées internes est un fait connu et s'explique par la continentalité croissante vers l'intérieur de la chaîne. La carte de MÜLLER et al. (1976) montre également une nette inflexion des isolignes à hauteur des Alpes vaudoises, qui pourrait faire conclure à l'existence d'une tendance est-ouest au front des Alpes. Nos résultats montrent que ces différences ne sont pas dues à des causes climatiques mais à des différences morphologiques dues au style tectonique des massifs montagneux.

Ainsi l'ensemble des Alpes calcaires occidentales suisses peut être considéré comme climatiquement homogène, et la répartition actuelle des glaciers y est expliquée de manière satisfaisante par un modèle régional unique.

\section{Remerciements}

Nous remercions le Prof. G. Furrer et le Dr Max Maisch pour la lecture attentive du manuscrit et pour leurs propositions d'amélioration.

La présente recherche a été soutenue par le Fonds national suisse de la recherche scientifique, requêtes no 21-27879.89 et $21-27874.89$.

\section{Références}

Levés originaux de la carte Dufour (archives Office fédéral de topographie):

Blatt XVII, Unterabt. 7. Original-Nr. 384. Levé par Bétemps et Wolfsberger, 1840. 1:50 000. (Correspond à Atlas Siegfried no 472.)

Blatt XVII, Unterabt. 8. Original-Nr. 385. Levé par Müller et Wolfsberger, 1841. 1:50 000. (Correspond à Atlas Siegfried no 473.)

Blatt XVII, Unterabt. 10. Original-Nr. 391 a. Levé par Wolfsberger, 1839. 1:50000. (Correspond à Atlas Siegfried no 477.)

BlattXVII, Unterabt. 11. Original-Nr. 399. Levé par Wolfsberger, 1839. 1:50 000. (Correspond à Atlas Siegfried no 481.)

Blatt XVII, Unterabt. 13. Original-Nr. 402 a. Levé par Müller, 1840. 1:50 000. (Correspond à Atlas Siegfried no 483.)

Blatt XVII, Unterabt. 14. Original-Nr. 406 a. Levé par Wolfsberger, 1840. 1:50000. (Correspond à Atlas Siegfried no 485.)

Blatt XXII, Section 1. Original-Nr. 475. Levé par L'Hardy, 1857. 1:50 000. (Correspond à Atlas Siegfried no 525.) 
Ouvrages et articles:

BLESS, R. (1984): Beiträge zur spät- und postglazialen Geschichte der Gletscher im nordöstlichen Mont-Blanc-Gebiet. Phys. Geogr. 15, Zürich.

DORTHE-MONACHON, C. (1986): Contribution à l'étude de la morphologie glaciaire de la vallée de l'Arve (Haute-Savoie, France). Thèse, Institut de Géographie, Université de Lausanne.

DORTHE-MONACHON, C. (1993): Etude des stades tardiglaciaires des vallées de la rive droite du Rhône entre Loèche et Martigny. Institut de Géographie Lausanne, Travaux et Recherches no 10.

ESCHER, A., MASSON, H., STECK, A. (1988): Coupes géologiques des Alpes occidentales suisses, Mémoire de Géologie no 2, Lausanne.

GROSS, G., KERSCHNER, H., PATZELT, G. (1977): Methodische Untersuchungen über die Schneegrenze in alpinen Gletschergebieten. In: Zeitschrift für Gletscherkunde und Glazialgeologie, 12/2, 223-251.

JEGERLEHNER, J. (1902): Die Schneegrenze in den Gletschergebieten der Schweiz. In: Gerlands Beiträge zur Geophysik, vol. V, 486-566.

LICHTENECKER, N. (1936): Die gegenwärtige und die eiszeitliche Schneegrenze in den Ostalpen, Verhandlungen der III. Int. Quartärkonferenz, Wien.

MAISCH, M. (1981): Glazialmorphologische und gletschergeschichtliche Untersuchungen im Gebiet zwischen Landwasser- und Albulatal (Kt. Graubünden, Schweiz), Phys. Geogr. 3, Zürich.

MAISCH, M. (1982): Zur Gletscher- und Klimageschichte des alpinen Spätglazials. In: Geogr. Helv. 37 /2, 93-104.

MAISCH, M. (1987 a): Die Gletscher um “1850" und "HEUTE" im Bündnerland und in den angrenzenden Gebieten: Untersuchungen zur Höhenlage, Veränderung und räumlichen Struktur von Schneegrenzen. In: Geogr. Helv. 42/2, 127-145. MAISCH, M. (1987 b): Zur Gletschergeschichte des alpinen Spätglazials: Analyse und Interpretation von Schneegrenz- daten. In G. FURRER et al:: Zur Gletscher-, Vegetations- und Klimageschichte der Schweiz seit der Spätzeit, Geogr. Helv. 42/2, 63-71.

MAISCH, M. (1988): Die Veränderungen der Gletscherflächen und Schneegrenzen seit dem Hochstand von $1850 \mathrm{im}$ Kanton Graubünden (Schweiz). In: Z. Geomorph. N. F., Suppl.-Bd. 70, 113-130.

MAISCH, M. (1992): Die Gletscher Graubündens, Phys. Geogr. 33, Zürich.

MONACHON, C. (1978): Essai de reconstitution de la paléogéographie des stades glaciaires de la Haute Sarine entre la crête des Hautes-Alpes calcaires et Gstaad. Mémoire Institut de Géographie, Université de Lausanne.

MÜLLER, F., CAFLISCH, T., MÜLLER, G. (1976): Firn und Eis der Schweizer Alpen. Gletscherinventar, ETH - Geographisches Institut, Publ. Nr. 57, Zurich.

NOVERRAZ, F. (1970): Etude géologique du versant français de la chaîne Pic de Tenneverge-Mont Ruan-Dents Blanches de Champéry. Diplôme Institut de Géologie, Université de Lausanne.

PHILLIPS, M. (1993): Géomorphologie du Vallon de Nant. Mémoire Institut de Géographie, Université de Lausanne.

REYNARD, E. (1992): Géomorphologie de la vallée de la Morge (Valais). Mémoire Institut de Géographie, Université de Lausanne.

SCHOENEICH, P. (en préparation): Le retrait glaciaire dans les vallées des Ormonts, de l'Hongrin et de l'Etivaz. Thèse, Institut de Géographie, Université de Lausanne.

STECK, A., EPARD, J.-L., ESCHER, A., MARCHAND, R., MASSON, H., SPRING, L. (1989): Coupe tectonique horizontale des Alpes centrales, Mémoire de Géologie no 5, Lausanne.

STUBER, A. (1989): La région de la Gemmi: géomorphologie, histoire et dynamique glaciaire. Mémoire Institut de Géographie, Université de Lausanne.

WETTER, W. (1987): Spät- und postglaziale Gletscherschwankungen im Mont-Blanc-Gebiet: untere Vallée de Chamonix-Val Montjoie, Phys. Geogr. 22, Zurich. 